\title{
Optimal operation of a pumped-storage hydro plant that compensates the imbalances of a wind power producer
}

\author{
Álvaro Jaramillo Duque ${ }^{\mathrm{a}, *}$, Edgardo D. Castronuovo ${ }^{\mathrm{a}}$, Ismael Sánchez ${ }^{\mathrm{b}}$, Julio Usaola ${ }^{\mathrm{a}}$ \\ ${ }^{a}$ Department of Electrical Engineering, Carlos III University of Madrid, Av. Universidad 30 - PC 28911, Leganés, Spain \\ ${ }^{b}$ Department of Statistic, Carlos III University of Madrid, Av. Universidad 30 - 28911, Leganés, Spain
}

\begin{abstract}
The participation of wind energy in electricity markets requires providing a forecast for future energy production of a wind generator, whose value will be its scheduled energy. Deviations from this schedule because of prediction errors could imply the payment of imbalance costs. In order to decrease these costs, a joint operation between a wind farm and a hydro-pump plant is proposed; the hydro-pump plant changes its production to compensate wind power prediction errors. In order to optimize this operation, the uncertainty of the wind power forecast is modeled and quantified. This uncertainty is included in an optimization problem that shifts the production of the hydro-pump plant in an optimal way, aiming at reducing the imbalance costs. The result of such a method is profitable for both participants, the wind farm and the hydro-pump plant. A realistic test case is used to evaluate the proposed method.
\end{abstract}

Keywords: Wind power, Management of forecast errors, Pumping storage, Wind-hydro coordination, Wind power scenarios, Optimization

\section{Nomenclature}

The following describes the nomenclature used for various variables and parameters throughout the paper. Boldface is used to denote vectors and matrices.

\section{Statistical parameters}

$\hat{\boldsymbol{\alpha}} \quad$ Estimated alpha, parameter of the beta distribution.

$\hat{\boldsymbol{\beta}} \quad$ Estimated beta, parameter of the beta distribution.

$\hat{\boldsymbol{\mu}} \quad$ Estimate conditional mean of the simulated wind power production.

$\hat{e} \quad$ Predicted errors of the wind power production.

$\hat{\boldsymbol{p}} \quad$ Prediction of the wind power production.

$\hat{\boldsymbol{v}}^{2} \quad$ Estimate conditional variance of the simulated wind power production.

$\hat{\sigma} \quad$ Standard deviation of the predicted errors.

e Calculated errors of the wind power production.

$\boldsymbol{p} \quad$ Wind power production.

$\boldsymbol{p}^{(s)} \quad$ Simulated wind power production.

\footnotetext{
${ }^{*}$ Corresponding author

Email addresses: alvaro.jaramillo@uc3m.es (Álvaro Jaramillo Duque), castronuovo@ieee .org (Edgardo D. Castronuovo), ismael@est-econ.uc3m.es (Ismael Sánchez), jusaola@ing.uc3m.es
} (Julio Usaola) $\boldsymbol{u}^{(s)}$

Cumulative distribution function of simulated prediction error.

$Y_{h} \quad$ Cumulative distribution function of wind power production, transitional state in the validation process.

$\Phi(\cdot) \quad$ Cumulative distribution function of the standard normal distribution.

$\Sigma \quad$ Covariance matrix of the prediction errors.

$E^{(s)} \quad$ Estimated energy imbalances of the wind power production.

$F_{h} \quad$ Beta cumulative distribution function for horizon $h$.

$h \quad$ Prediction horizon, discretized periods in hours.

$P \quad$ Multivariate cumulative density function of the wind power production.

$s \quad$ Simulated scenarios.

$t \quad$ Time, discretized periods in hours.

\section{Optimization problem}

$\bar{P}_{\mathrm{H} i} \quad$ Scheduled hydraulic action, power generation.

$\bar{P}_{\mathrm{P} i} \quad$ Scheduled pump action, power consumed.

$\eta_{\mathrm{H}} \quad$ Generator and hydraulic reservoir efficiency.

$\eta_{\mathrm{P}} \quad$ Pump and pipes efficiency.

$\hat{P}_{\mathrm{W} i} \quad$ Prediction of the wind power production. 
$c_{i}^{\text {buy }} \quad$ Reserve energy buy price.

$c_{i}^{\text {sell }} \quad$ Reserve energy sell price.

$c_{i} \quad$ Energy hourly price in the market.

$c_{\mathrm{P} i} \quad$ Hourly cost of the pumping operation.

$E_{i}^{\mathrm{R}} \quad$ Required energy store capacity in the reservoir.

$E_{i}^{\mathrm{S}} \quad$ Required minimum energy stored in the reservoir.

$E^{u} \quad$ Upper limit of energy stored in the reservoir.

$E_{i} \quad$ Storage energy in the reservoir.

$i \quad$ Hourly discretization periods.

$P_{\mathrm{H}}^{u} \quad$ Upper operation limit of the generator.

$P_{\mathrm{P}}^{u} \quad$ Upper operation limit of the pump.

$P_{\mathrm{W} i}^{M} \quad$ Maximum positive imbalance of the wind power producer.

$P_{\mathrm{W} i}^{m} \quad$ Maximum negative imbalance of the wind power producer.

$P_{\mathrm{H} i} \quad$ Power produced by the hydro-pump plant.

$P_{\mathrm{P} i} \quad$ Power consumed by the plant operating as a pump.

$P_{\mathrm{W} i} \quad$ Power produced by wind farm.

\section{Introduction}

In recent years, wind power generation has increased all over the world, mainly in Europe and the USA. The integration of wind power generation in electric power systems needs to be carefully performed and requires new concepts in operation, control and management. As the wind production at a given instant in the future can only be estimated, the integration of wind farms involves new uncertainties in system operation. In order to attain optimal management of the system, it is necessary to include the information on uncertainty of wind power predictions, as well as the use of optimizations tools.

Wind speed prediction was until recently only considered for weather analysis. Now, the high level of penetration of wind generation in electric systems has resulted in the increased importance of this prediction, because of the significant economic and technical impact resulting from erroneous generation predictions. Generally wind farms inject into the system all the available wind power obtainable from the wind, since the primary resource (the wind) has operational costs near zero. Therefore, an imbalance in the expected wind power production may significantly modify the market operation, requiring additional power reserves in the system operation. For this reason, new tools are necessary to provide reliable information about wind power production [1] and to compensate the probable imbalances in wind power generation (as proposed in the present paper).
Some works have analyzed prediction methods for wind power forecasts. Ref. [2] studies an area with high wind power penetration, aiming to quantify the necessary reserves to ensure the proper functioning of the electrical system. In this case, the forecasting error for wind power generation is expressed as an equation based on the prediction horizon, using the standard imbalance of the errors. This estimation is based on real data from a wind farm, with results obtained from a fuzzy calculation tool. Additionally, to obtain the errors in an area with many wind farms, a correlation function based on the distance between the different wind farms is used. In [3], a function that estimates the possible errors is proposed. It uses the time horizon as the only predictor variable, based on the average of different methods to predict the errors in wind production. Another proposal has been made in [4], where the wind prediction is represented as an ARMA series, then converting this data into electrical power using the curves of the wind generators.

An analysis of errors in wind power production shows dependence not only on the prediction horizon, but also on the predicted power level [5]. This concept is presented and applied in $[6,7]$, modeling wind power production as a non-parametric distribution.

The use of storage to compensate wind power imbalances has been studied in recent years. In [8], the collaboration between a wind farm and a hydro-pump plant, to reduce the economic losses caused by operational restrictions, was proposed. Considerations about the optimal size of the wind farm and the elements of the hydro-pump plant were made in [9]; the main object of these works was to use the hydro storage facility to increase the controllability of the wind farm and additionally maximize revenues. In [3], the utilization of a generic energy storage device for balancing the differences between forecasted and real productions in a wind farm is analyzed, when acting in a market environment. Cooperation between a wind farm and a conventional multi-reservoir hydropower system is considered in [4], in order to avoid the congestion on the adjacent transmission lines for a problem analyzed during the course of one year. In [10], the requirements of reserve in energy systems with large wind power penetration is calculated, minimizing the operation costs. A method to optimize the storage size for remote communities with wind and diesel generation is developed in [11]. The combined operation of wind farms and a pump hydro facility is analyzed in [12], considering the uncertainties in both wind power generation and market prices. In [13], some of the tools for the effective integration of large amounts of wind energy in the system, including the utilization of storage devices, are also considered. Two methods for minimizing the penalties due to imbalances of the wind farm power output are proposed in [14]; the first one considers the wind farm to bid alone in the day-ahead market, trying to minimize the risk of the bid and based on a statistical analysis of the expected production probability; the second one couples a hydro power plant with the wind farm, in order to minimize the imbalance costs incurred by the wind farm owner.

Most of the previous works analyze the storage action as internal (or strongly related) to wind power production, exclusively using the storage ability to compensate the wind power 
imbalances. However, this approach is not representative for large pumped stations in power systems. The present work aims to calculate the best operation of a storage plant, simultaneously following two objectives: a) to maximize the revenue in the conventional operation of the storage plant (as in a dailybased conventional procedure); and b) to offer a reserve to a wind power producer for managing the power imbalances. The required reserve depends on the accuracy of the wind power forecasts, which is unknown in advanced. Therefore, a statistical method to estimate a sequence of hourly expected forecasting errors given a sequence of hourly wind power predictions is developed. The proposed statistical method can be applied to wind power predictions coming from any prediction tool and allows the calculation of probable wind power productions scenarios in the near future. The proposed technique is applied to a real hydro-pump plant and actual data from a wind farm in the northwest of Spain.

The remainder of this paper is organized as follows. Section 2 describes a model and simulation procedure to estimate the uncertainty of the wind power production based on its previous predictions. Section 3 presents an optimization problem (introducing the uncertainty of the wind power prediction as an input) to obtain the best operation of a hydro-pump plant and compensating the possible imbalances of a wind power producer. In Section 4, the result of programmed operation and the real operation of the hydro-pump plant is analyzed and compared. Then, a similar assessment is done, but using different scenarios of wind production and energy prices as test cases. Finally, in Section 5, conclusions are duly drawn.

\section{Simulating Scenarios and Prediction Intervals in Wind Power Forecasting}

\subsection{Proposed Methodology}

A methodology for the simulation of statistical scenarios for wind power production in a short term horizon (one or two days in advance) is proposed here. The methodology for the simulation of scenarios is based on the so-called gaussian copula function $[15,16]$, that takes into account both the properties of univariate h-step ahead density forecast and the properties of the joint multivariate distribution function of multi-step ahead prediction errors. With the information provided by the calculated scenarios, both energy imbalances for some periods and prediction intervals for each hour are estimated [17]. This information is used to estimate uncertainty in wind power production and to optimize decisions depending on such uncertainty.

Let $\boldsymbol{p}_{t}$ be the observed wind power at time $t$ and let $\hat{\boldsymbol{p}}_{t+h \mid t}$ be the prediction of $\boldsymbol{p}_{t+h}$ made at time $t$. Since the future production $\boldsymbol{p}_{t+h}$ is still unknown at time $t$, it is represented as a random variable $\boldsymbol{P}_{t+h \mid t}$ with multivariate cumulative density function

$$
F_{P}\left(p_{t+1} ; \ldots ; p_{t+H}\right)=P\left(P_{t+1 \mid t}<p_{t+1} ; \ldots ; P_{t+H \mid t}<p_{t+H}\right) .
$$

The actual values $p_{t+1}, \ldots, p_{t+H}$, can then be considered as a realization (trajectory) of the multivariate random variable
$\boldsymbol{P}_{t+h \mid t}=\left(P_{t+1 \mid t}, \ldots, P_{t+H \mid t}\right)^{T}$. Similarly, the prediction errors obtained using the prediction $\hat{\boldsymbol{p}}_{t+h \mid t}$ are also unknown at time $t$ and, hence, should also be treated as a vector of random variables, defined as:

$$
e_{t+h \mid t}=\left[e_{t+1 \mid t}, e_{t+2 \mid t}, \cdots, e_{t+H \mid t}\right]^{T},
$$

with $T$ being the transpose operator, and with

$$
\boldsymbol{e}_{t+h \mid t}=\boldsymbol{P}_{t+h \mid t}-\hat{\boldsymbol{p}}_{t+h \mid t} .
$$

The wind power scenarios can be interpreted as random trajectories from $\boldsymbol{P}_{t+h \mid t}$. In order to generate these trajectories, it is necessary to know the multivariate distribution function, which can be complex due to the non linearity of the series. Whereas the multivariate random variable $\boldsymbol{P}_{t+h \mid t}$ can be complex, the univariate marginal distribution of $P_{t+h \mid t}$ for a given horizon can be easier to handle. In particular, it is well known that the distribution of $P_{t+h \mid t}$ conditional to the available prediction $\hat{p}_{t+h \mid t}$ is a bounded random variable with a conditional variance and skewness that depends on $\hat{p}_{t+h \mid t}([5,18,19,20])$, and with a shape that can be approximated with a beta distribution [21]. In this article the beta distribution, with cdf denoted as $F_{h}\left(p_{t+h} ; \alpha_{t, h}, \beta_{t, h}\right)$, is also used. The parameters $\alpha_{t, h}$, $\beta_{t, h}$ of the beta distribution are time varying. For a given period $t$ and horizon $h$ they are estimated using their relationship with the conditional mean and variance of $P_{t+h \mid t}$ as explained below. Given a set of marginal distributions $F_{1}\left(p_{t+1} ; \alpha_{t, 1}, \beta_{t, 1}\right)$, ..., $F_{H}\left(p_{t+H} ; \alpha_{t, H}, \beta_{t, H}\right)$, the multivariate distribution $F_{P}$ is obtained using a so-called copula function. Let the standard uniform random variables $U_{h}=F_{h}\left(P_{t+h \mid t}\right), h=1, \ldots, H$, then, the multivariate distribution function $C$ of $\left(U_{1}, \ldots, U_{H}\right)$ is called a copula. A copula is then a multivariate function, $C\left(u_{1}, \ldots, u_{h}\right)$, that 'couple' multivariate distributions to their one-dimensional marginal distribution. According to Sklar's theorem [22], there exist a copula $C$ that

$F_{P}\left(p_{t+1} ; \ldots ; p_{t+H}\right)=C\left[F_{1}\left(p_{t+1} ; \alpha_{t, 1}, \beta_{t, 1}\right), \ldots, F_{H}\left(p_{t+H} ; \alpha_{t, H}, \beta_{t, H}\right)\right]$

Conversely, if $C$ is a copula and $F_{1}, \ldots, F_{H}$ are univariate distribution functions, then $F_{P}$ defined by (4) is a joint distribution function with marginals $F_{1}, \ldots, F_{H}$. In this way the multivariate distribution $F_{P}$ is decomposed into the marginal distribution functions and a copula. Besides, the marginal distribution functions and the copula can be estimated separately. This result can be used to simulate a trajectory of $\boldsymbol{P}_{t+h \mid t}$ by simulating a trajectory $\left(u_{1}, \ldots, u_{H}\right)$ of the copula function and then using the transformation $F_{i}^{-1}\left(u_{i}\right)$ to transform the uniform variables $u_{i}, i=1, \ldots, H$, to attain the desired marginal distributions. The choice of the copula function depends on the particular application, and its validity should be evaluated with the data. In our case, the selection of the copula function is based on the properties of the prediction errors. From (3) it can be seen that the randomness of $\boldsymbol{P}_{t+h \mid t}$ comes from $\boldsymbol{e}_{t+h \mid t}$. The properties of $\boldsymbol{e}_{t+h \mid t}$ can be estimated with a set of historical errors, denoted as

$$
\hat{e}_{t+h \mid t}=\left[\hat{e}_{t+1 \mid t}, \hat{e}_{t+2 \mid t}, \cdots, \hat{e}_{t+H \mid t}\right]^{T},
$$


where $\hat{e}_{t+h \mid t}=\boldsymbol{p}_{t+h}-\hat{\boldsymbol{p}}_{t+h \mid t}$.

The data from the case study below reveals that the marginal unconditional distribution of these prediction errors for each horizon could well be approximated by a normal distribution with zero mean. This normality is, in addition, a usual assumption for the error term of statistical models [23]. This result suggests the selection of the gaussian copula, whose covariance matrix $\Sigma$ needs to be estimated. The estimation of $\Sigma$ is made assuming that the random vector of prediction errors $e_{t+h \mid t}$ follows a multivariate normal distribution of zero mean and covariance matrix:

$$
\Sigma=\left(e_{t+h \mid t} e_{t+h \mid t}^{T}\right)
$$

which, assuming second order stationarity, can be estimated from the historical data. It should be remarked that in a general application of the gaussian copula, this underline function might not be observed. Then, $\Sigma$ should be estimated from the pairwise comparison of the non-gaussian marginal distributions. Typically, this is done by computing a rank correlation matrix (e.g. Kendall's tau or Spearman rho coefficients) of the marginals distributions and then transforming it into a linear correlation matrix $R$. For instance, it is well known that the relationship between the Spearman rho, $\rho_{s}$, and the linear correlation coefficient, $\rho$, in a bivariate normal distribution is $\rho=2 \cdot \sin \left(\rho_{s} \cdot \pi / 6\right)$. The estimate $\Sigma$ is then computed by multiplying the correlations by the product of the sampling standard deviation of the unconditional prediction errors at each horizon, denoted as $\sigma_{h}, h=1,2, \ldots, H$. This approach could also be applied here. The estimation of $\Sigma$ using (6) is, however, computationally more convenient. For instance, it easily allows for time-varying estimation procedures like in [24].

Given a sequence of power forecasts, $\hat{\boldsymbol{p}}_{t+h \mid t}, h=1, \ldots, H$, a statistical scenario (trajectory) of future wind power production can be computed with the gaussian copula following a two-step procedure. In the first step, a realization of $e_{t+h \mid t}, h=1, \ldots, H$ is obtained by a random generation from a normal distribution of zero mean and covariance matrix $\Sigma$. This sequence of simulated prediction errors is denoted as $\boldsymbol{e}_{t+h \mid t}^{(s)}=\left[e_{t+1 \mid t}^{(s)}, e_{t+2 \mid t}^{(s)}, \cdots, e_{t+H \mid t}^{(s)}\right]$, $s=1, \ldots, S$, where $S$ is the number of simulated scenarios. Since the unconditional distribution of prediction errors is assumed normal, the covariance matrix contains all the information about the interdependence of the forecasting errors.

In the second step, each simulated trajectory of prediction errors is transformed into a simulated trajectory of power productions, denoted as $\boldsymbol{P}_{t+h \mid t}^{(s)}=\left(p_{t+1 \mid t}^{(s)}, \ldots, p_{t+H \mid t}^{(s)}\right)^{T}$. To perform this transformation, and as mentioned above, the marginal distribution of $P_{t+h \mid t}$ conditional to the available prediction $\hat{p}_{t+h \mid t}$ is used. By doing that, a distribution with lower variance than the unconditional distribution is used and, consequently, the uncertainty of the predictions is reduced. These conditional marginal distributions are modelled as beta distributions. This transformation is made as follows. First, the trajectory $e_{t+h \mid t}^{(s)}$ is transformed into a trajectory of uniform random variables in the unit interval by applying to each simulated prediction error $\boldsymbol{e}_{t+h \mid t}^{(s)}$ the cumulative distribution function (cdf) of the normal distribu- tion, obtaining $\boldsymbol{u}_{t+h \mid t}^{(s)}$. That is:

$$
\boldsymbol{u}_{t+h \mid t}^{(s)}=\Phi\left(\frac{\boldsymbol{e}_{t+h \mid t}^{(s)}}{\hat{\sigma}_{h}}\right) \quad \forall h,
$$

where $\Phi(\cdot)$ is the cdf of the standard normal distribution, and $\hat{\sigma}_{h}$ is the sampling estimate of the square root of the h-th element of diagonal of the covariance matrix (6). Second, each element $\boldsymbol{u}_{t+h \mid t}^{(s)}$ is transformed into a $\boldsymbol{p}_{t+h \mid t}^{(s)}$ by applying the inverse of the corresponding beta distribution. That is,

$$
\boldsymbol{p}_{t+h \mid t}^{(s)}=F_{h}^{-1}\left(\boldsymbol{u}_{t+h \mid t}^{(s)} ; \hat{\boldsymbol{\alpha}}_{t, h}, \hat{\boldsymbol{\beta}}_{t, h}\right)
$$

where $F_{h}$ is the cdf of the beta distribution with estimated parameters $\hat{\boldsymbol{\alpha}}_{t, h}$ and $\hat{\boldsymbol{\beta}}_{t, h}$. These estimates are obtained using the relationship of these parameters with the mean and the variance of the distribution as:

$$
\begin{gathered}
\hat{\boldsymbol{\alpha}}_{t, h}=\hat{\boldsymbol{\mu}}_{t, h}\left(\frac{\hat{\boldsymbol{\mu}}_{t, h}\left(1-\hat{\boldsymbol{\mu}}_{t, h}\right)}{\hat{\boldsymbol{v}}_{t, h}^{2}}-1\right) \quad \forall h, \\
\hat{\boldsymbol{\beta}}_{t, h}=\left(1-\hat{\boldsymbol{\mu}}_{t, h}\right)\left(\frac{\hat{\boldsymbol{\mu}}_{t, h}\left(1-\hat{\boldsymbol{\mu}}_{t, h}\right)}{\hat{\boldsymbol{v}}_{t, h}^{2}}-1\right) \quad \forall h,
\end{gathered}
$$

where $\hat{\boldsymbol{\mu}}_{t, h}$ is an estimate of the conditional mean of $\boldsymbol{p}_{t+h \mid t}^{(s)}$, and $\hat{\boldsymbol{v}}_{t, h}^{2}$ an estimate of its conditional variance. The conditional mean is estimated with expected production, i.e. $\hat{\boldsymbol{\mu}}_{t, h}=\hat{\boldsymbol{p}}_{t+h \mid t}$. The conditional variance of this distribution is usually estimated using a polynomial function of the prediction, [5], using binaggregated data for each horizon. To compute this estimate, data have been grouped according to the prediction level using bins of 0.02 [pu] of predicted power (the last interval is larger because the operating range of the wind farm usually does not exceed $85 \%$ of capacity). In each bin, the average prediction and its variance is computed. Then a fourth-order polynomial function of the prediction is estimated by least squares. Fig. 1 illustrates this estimation for $h=28$ using the data of the case study.

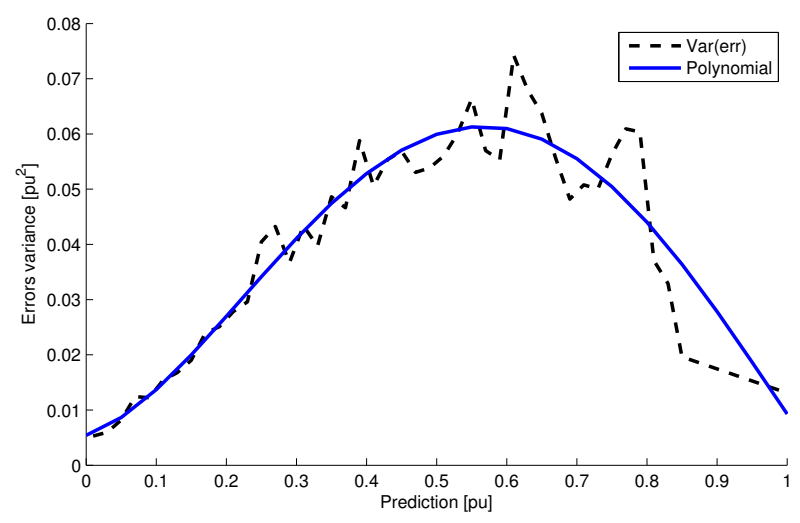

Figure 1: Variance function of the errors, considering the level of prediction power and the horizon for $h=28$. 


\subsection{Case Study}

The data set used to illustrate the proposed methodology consists of the hourly wind power production of a wind farm located in the northwest of Spain during a two year period. Hourly wind power predictions for up to $H=35$ hours ahead are available for this period. The predictions were obtained from an efficient forecasting tool based on statistical models that uses as input data both predictions of wind speed and direction as well as on-line measures of the real hourly wind power generated in the wind farm $[25,24]$. The data is normalized to the rated capacity of the wind farm for statistical analyses.

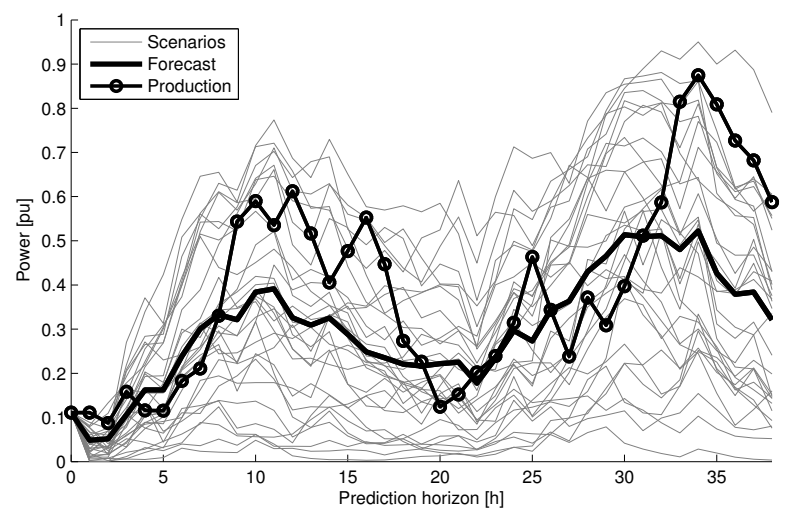

Figure 2: Possible scenarios, production and prediction of wind power.

Fig. 2 shows only thirty of the calculated probable scenarios, $\boldsymbol{p}_{t+h \mid t}^{(s)}$, and the wind power prediction, $\hat{\boldsymbol{p}}_{t+h \mid t}$. Each scenario follows a path that inherits the sequence of the errors, which means that each point of the scenario depends on its previous value, the estimated covariance of errors, and the wind power prediction.

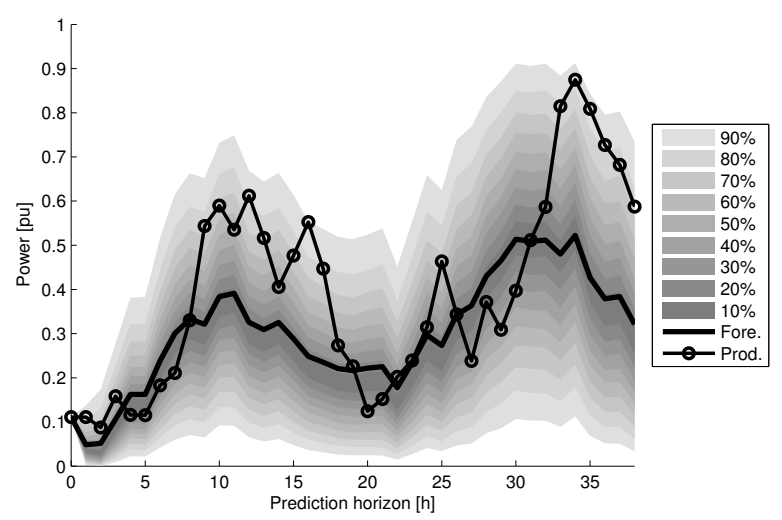

Figure 3: Prediction intervals of the scenarios.

Fig. 3 plots the prediction intervals obtained from all the simulated scenarios, represented in bands; in addition, the wind power forecasting, $\hat{\boldsymbol{p}}_{t+h \mid t}$ and the power production, $\boldsymbol{p}_{t+h}$ are drawn. Each band represents the probability that the wind power production has to be within the range covered by this probability area at future time $h$. The broadest band corresponds to $90 \%$. Bands with more narrow margins are shown inside it.

The main advantage of the proposed methodology is the simplicity in achieving the simulated scenarios and the ability to quantify the uncertainty in the wind power predictions, taking into account the relationship between the errors at different time horizons and the conditional behavior of the wind power prediction.

\subsection{Energy Imbalances}

Due to the uncertainty in the wind power forecast for the near future, it is necessary to reserve an amount of energy to cover the energy imbalance between programmed and real productions. In some cases, the amount of energy reserved for this purpose is generally larger than necessary and calculated by using heuristic methods, with the intention of avoiding possible operation problems. However, these strategies may be far from optimal.

The advantage of the proposed method to simulate scenarios is in the capacity to estimate, for each simulated scenario, the energy imbalance $E^{(s)}$. This imbalance is computed in the time interval $\left[h_{a}, h_{b}\right]$, as the integral of the difference between the wind power forecast and each wind simulated scenario, as in (11). Using a large number of scenarios, it is possible to construct the distribution of these imbalances. This distribution informs about the probability of incurring into different levels of energy imbalances.

$$
E^{(s)}=\int_{h_{a}}^{h_{b}}\left(\hat{\boldsymbol{p}}_{t+h \mid t}-\boldsymbol{p}_{t+h \mid t}^{(s)}\right) d h \quad \forall s
$$

\subsection{Validation of the Simulated Scenarios of Wind Power Pro- duction}

The evaluation of the simulated scenarios has two parts, both related with the design of the copula: the evaluation of the conditional marginal distribution and the evaluation of the dependence of the sequence of simulated trajectories. First, the use of the beta distribution to model the marginal distribution of power production is evaluated. This is made by applying (8) inversely. That is, if a given beta distribution of parameters (9) and (10) is appropriate to model the conditional distribution of $\boldsymbol{p}_{t+h}$, then

$$
\boldsymbol{Y}_{h}=F_{B}\left(\boldsymbol{p}_{t+h} \mid \hat{\boldsymbol{\alpha}}_{t, h}, \hat{\boldsymbol{\beta}}_{t, h}\right) \quad \forall h
$$

will follow a uniform random variable. This evaluation technique is known as the Probability Integral Transform (PIT). We have applied the inverse transformation (12) to all historical wind power production and to all horizons, using at each time the corresponding beta distribution with estimated parameters (9) and (10). The result is shown in the histogram in Fig. 4. A dashed horizontal line marks the ideal result of a perfect uniform histogram. The distribution of data obtained is quite good, even compared to a case obtained from a uniform random number generator. This confirms that the beta distribution conditional to the predicted power is a good assumption to model the predictive density of wind power production. 


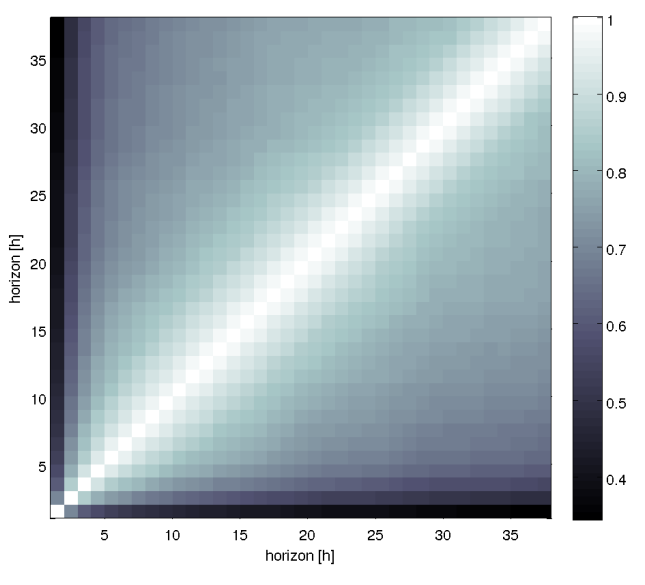

(a) Spearman ranks $\boldsymbol{e}$.

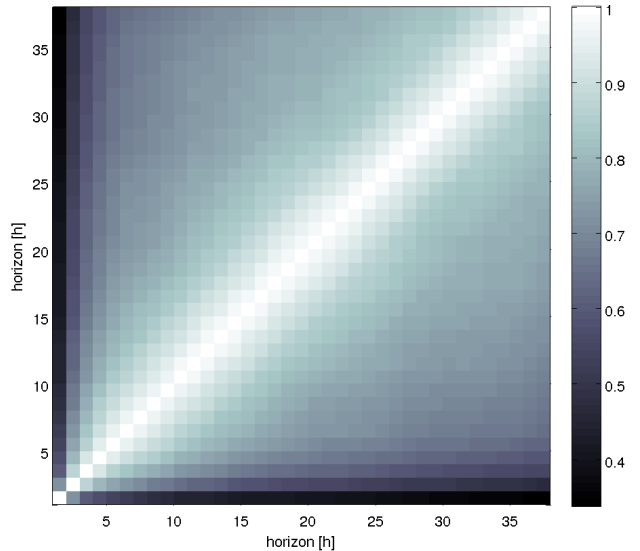

(b) Spearman ranks $\boldsymbol{e}^{(s)}$.

Figure 5: Rank correlation of the errors on the predictions and the errors on the simulated trajectories.

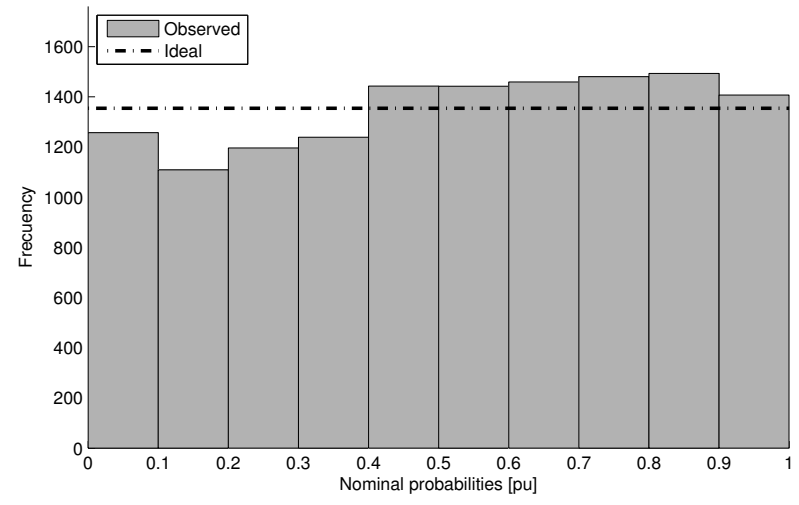

Figure 4: Validation of the proposed method, marginal distribution.

The second evaluation exercise consist of verifying that the dynamic dependence of the simulated trajectories is similar to the dynamic of actual production data. To this aim, the rank correlations coefficient of Spearman is used. This rank correlation coefficient measures the degree to which large or small values of one variable are associate with large or small values of a second one. When marginal random variables are not gaussian, linear correlation coefficient might prove very misleading. However, rank correlation coefficients are robust to this lack of normality and are more appropriate. In order to compare the actual data and the simulated trajectories we compute the rank correlation matrix with the pairwise comparison of prediction errors at alternative horizons. We compare the rank correlation matrix using actual prediction errors with the one based on the simulated trajectories. These matrices are shown in Fig. 5(a) and 5(b). It can be seen that both matrices are very similar. A matrix containing the difference between these two matrices is computed and the maximum, minimum, average and standard deviation of the values of this difference matrix is obtained. These values are: $0.0204,-0.0225,0.0057$, and 0.0007 respec- tively. These values confirm that the simulated trajectories have a dynamic dependence very similar to the real data.

\section{Optimal Operation of a Hydro-Pump Plant}

\subsection{Conventional Operation}

In order to analyze the optimal operation program of the hydro-pump plant considering typical reserve objectives, the conventional operation of a hydro-pump plant is at first formulated. In the present study, the daily operation is considered, with hourly discretization. A perfect forecast of energy prices in the next day market is assumed. However, short-term forecasting of energy prices [12, 26] could also be included in the analysis when necessary. The hydro-pump plant bids power quantities to the daily market, without considering intraday market corrections.

The objective of the operation is to maximize the daily revenue of the hydro-pump plant. The plant pumps water in a reservoir in low-price periods, working as a load, and then discharging the stored water during high-price periods, operating then as hydraulic generator $[8,9]$. The operation can be summarized in the following optimization problem. Hereafter we will refer to this problem as "independent operation" of the hydropump plant. 


$$
\begin{aligned}
\max & \sum_{i=1}^{n}\left(c_{i} \bar{P}_{\mathrm{H} i}-c_{\mathrm{P} i} \bar{P}_{\mathrm{P} i}\right) \\
\text { s. t. } & \\
E_{i+1} & =E_{i}+t\left(\eta_{\mathrm{P}} \bar{P}_{\mathrm{P} i}-\frac{\bar{P}_{\mathrm{H} i}}{\eta_{\mathrm{H}}}\right) \\
E_{1} & =E_{1}^{\exp } \\
E_{n+1} & =E_{n+1}^{\exp } \\
0 & \leq \bar{P}_{\mathrm{H} i} \leq P_{\mathrm{H}}^{u} \\
\bar{P}_{\mathrm{H} i} & \leq \eta_{\mathrm{H}} \frac{E_{i}}{t} \\
0 & \leq \bar{P}_{\mathrm{P} i} \leq P_{\mathrm{P}}^{u} \\
0 & \leq E_{i} \leq E^{u} \\
\forall & \quad i=1, \ldots, n
\end{aligned}
$$

In the notation, the variables of the anticipatory programming will be marked with a bar at the top, “-”. When the variable has no bar, this means that it is the actual value.

Objective function (13) is the difference between the revenue of selling the energy and the cost of pumping. The latter is the cost of buying energy on the market plus the internal cost of the operation.

In equation (14), the level of stored energy for the next period is calculated. This value depends on the pumping or generation actions and their efficiencies. The initial level of the reservoir is generally known, and the final level is obtained from the medium-term planning, equations (15) and (16) respectively. In the present case, both levels are set to the same value.

The amount of power injected into the network is determined by the physical capacity of the generator, (17). Additionally, it cannot be greater than the available energy in the reservoir considering the efficiency, equation (18). The amount of power consumed as a pump is also determined by the physical capacity of the machine, equation (19). Finally, the reservoir is limited by the physical capacity, (20). Additional technical restrictions can be included in the formulation to represent particular characteristics.

The study case takes the specifications from an actual Spanish hydro-pump plant that covers the imbalances of a wind power producer: $P_{\mathrm{H}}^{u}=32.8[\mathrm{MW}], P_{\mathrm{P}}^{u}=40.3[\mathrm{MW}]$, $E_{u}=240[\mathrm{MWh}], \eta_{\mathrm{H}}=88[\%]$ and $\eta_{\mathrm{P}}=92[\%]$. Taking as a base the wind farm power: $P_{\mathrm{W}}^{u}=30[\mathrm{MW}]$, the corresponding values in per-unit are: $P_{\mathrm{H}}^{u}=1.09[\mathrm{pu}], P_{\mathrm{P}}^{u}=1.34[\mathrm{pu}]$ and $E_{u}=8.00[\mathrm{pu}]$.

In Fig. 6, the result of the problem defined in the equations (13)-(20) is shown. In the figure, the operation of the plant as a pump, $\bar{P}_{\mathrm{P} i}$, the operation of the plant as hydraulic generator, $\bar{P}_{\mathrm{H} i}$, the level of energy stored in the reservoir, $E_{i}$ and the energy prices in the market are depicted, $c_{i}$. The price of energy is a determining factor in the outcome of the optimal schedule; a different profile of energy prices would modify the optimal program.

In the independent activity, the operation starts without any action on the plant, because the plant is waiting for lower prices before starting operating as a pump. In this period, the level of energy stored in the reservoir is $E_{1}^{\exp }$. When prices are low enough, the plant begins the operation as a pump, until it is full or obtains the optimum stored energy (from hour 2 at the end of hour 7). After that, the storage plant waits for higher prices. The hydro-pump plant sells the energy during the periods of highest prices. In the period between hours 17 and 23 , the hydro-pump plant operates as a generator and sells the stored energy to the market, until it reaches the final value of energy required in the reservoir, $E_{n+1}^{\exp }$. In the last hour of the optimal program, the plan does not perform any action and the energy level is maintained in the reservoir.

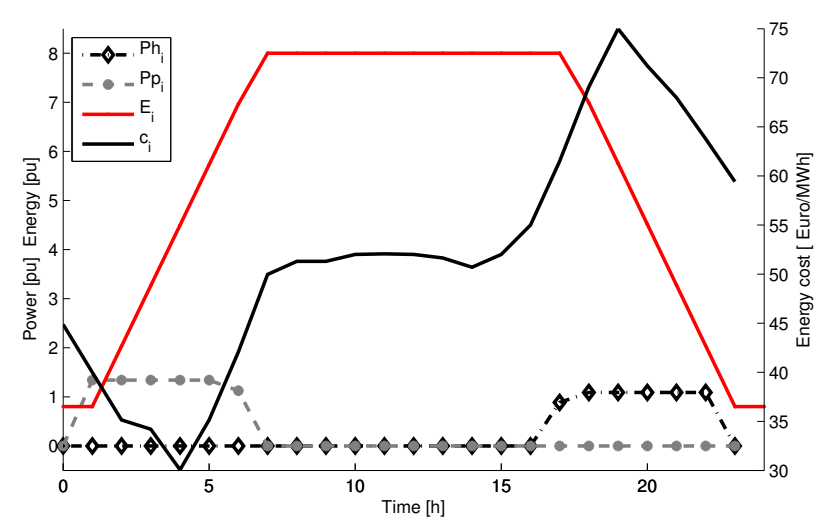

Figure 6: Optimal operation for a hydro-pump plant.

\subsection{Operation considering the imbalances in wind power pro- duction}

In the present section, the objective of the hydro-pump plant is to optimize the daily revenue of the plant and also cover the possible imbalances of wind power producers. Hence the hydro-pump plant simultaneously aims to: a) operate in the daily market, as usual, i.e., as if there were no wind generation; b) coordinate with the wind power producer, trying to partially compensate the wind power imbalances. According to objective b), the plant should adjust the operation at any time, either reducing or increasing the hydro generation, as well as the pumping operation, trying to cover the imbalances of the wind power producer. In both cases, from the system operation point-of-view, the wind power producer and hydro-pump plant would follow a program without errors (if the hydro-pump plant is contracted to cover all the wind power imbalances).

The original problem, equations (13)-(20), must be adapted to these new requirements. First, it is necessary to establish the magnitudes of the errors of wind power and energy previsions that must be covered by the hydro-pump plant. These values depend on two factors: a) the expected errors in the forecasted values of the wind power production and b) the assumed risk in the compensation of the imbalances. The expected errors are calculated in this paper using the method presented in Section 2 and [17]. As shown in Fig. 3, compensating 90\% of the probable power energy imbalances requires a large reserve of active power in the hydro-pump plant. By reducing the band (compensating only $80 \%$ or $70 \%$ of the probable scenarios) the 
wind power producer decreases the reserve contracted in the hydro-pump plant, but with the economic risk of compensating imbalance costs in the reserve market.

\subsubsection{Wind Power Imbalance}

In Spain, renewable generators must provide the market with predictions of the hourly production for the next day, 14 hours before the beginning of the day. The actual generation of the wind power producer is always different from the forecasted values. Any deviation from the schedule implies a cost. In the present case, it is considered that the wind power producer can buy capacity from the hydro-pump plant to compensate the imbalances in the production, instead of buying compensation from the reserve market.

When the wind power production is smaller than expected (as informed to the market in the scheduled operation program) and the hydro-pump plant is pumping, the lack of generation can be easily compensated by reducing the pump action (reducing the load in the same quantity). Alternatively, if the hydro-pump plant is acting as hydro generator, the action of compensation requires additional power generation. Therefore, the hydro-pump plant must always reserve an amount of power generation to compensate probable wind power underproduction; hence, the maximum power generated in each period must be limited by $P_{\mathrm{H}}^{u}-P_{\mathrm{W} i}^{m}$.

By contrast, when wind power production is larger than scheduled and the hydro-pump plant operates as a pump, the hydro plant must be capable of storing the overproduction. For this reason, the maximum power consumed in each period in the hydro-pump plant must be $P_{\mathrm{P}}^{u}-P_{\mathrm{W} i}^{M}$. On the other hand, the overproduction in the wind power producer, when the storage plant is operating as hydro generator, can be easily compensated by reducing the hydro production.

In the inactive period between the filling and discharge of the reservoir, compensation actions may also be required by the wind power producer. These compensations are analyzed in the next section.

\subsection{Energy Storage Minima}

Two restrictive cases must be considered:

a) If the reservoir is almost full (periods 6-16 in Fig. 7) and the hydro-pump plant must compensate possible overproduction of the wind power producer, it is necessary to reserve storage capacity in the reservoir in each period. Therefore, the reservoir must not be filled to its entire capacity.

b) If the reservoir is at its minimum level and has to compensate probable underproduction of the wind power producer, a minimum reserve of energy in the reservoir must be maintained. Therefore, it is necessary to reserve a minimum amount of energy stored in the reservoir at the beginning of the period, when the plant is still waiting for lower prices, and at the end of the operation. The minimum of stored energy must be enough to compensate the imbalances of the wind producer and can be calculated by the accumulative addition of probable imbalances, as presented in Section 2. In the present case, $E_{1}^{\mathrm{esp}}$ and $E_{n+1}^{\text {esp }}$ are considered larger values than the minimum required by the accumulated errors.
The coverage level is adjusted at $90 \%$ of the simulated cases, both for positive imbalances (overproduction, where it is necessary to reserve a storage energy capacity) and for negative imbalances (underproduction, which requires a minimum amount of energy stored).

\subsection{Pre-operation program}

In the present section, the results of the pre-operation program, considering the reserves in power and energy, of the hydro-pump plant are shown. For these simulations, the optimization problem (13)-(20), is solved, substituting eq. (17), (19) and (20) by (21), (22) and (23). Hereafter, we will refer to this problem as "joint operation".

$$
\begin{gathered}
0 \leq \bar{P}_{\mathrm{H} i} \leq\left(P_{\mathrm{H}}^{u}-P_{\mathrm{W} i}^{m}\right) \\
0 \leq \bar{P}_{\mathrm{P} i} \leq\left(P_{\mathrm{P}}^{u}-P_{\mathrm{W} i}^{M}\right) \\
E_{i}^{\mathrm{S}} \leq E_{i} \leq\left(E^{u}-E_{i}^{\mathrm{R}}\right) \\
\forall \quad i=1, \ldots, n
\end{gathered}
$$

In Fig. 7, the result of the joint operation of the hydro-pump plant is presented, compensating $90 \%$ of the probable wind power imbalances, Fig. 3.

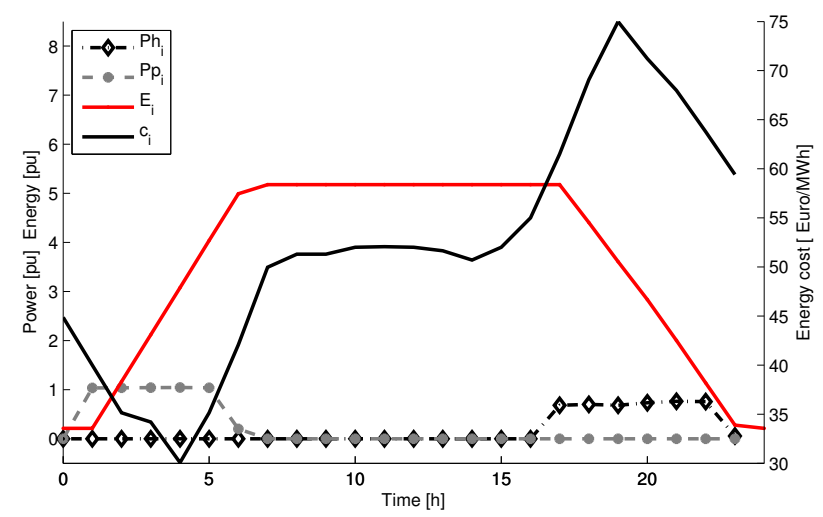

Figure 7: Optimal operation for a hydro-pump plant compensating $90 \%$ the imbalances for a wind power producer.

Comparing Fig. 7, joint operation, with Fig. 6, independent operation, it is possible to observe a reduction in the levels of operation a pump and as a generator, and also a reduction in the energy stored in the reservoir. This is due to restrictions imposed by the possible imbalances, positive and negative, of the wind power producer and the capability to store energy, equations (21), (22) and (23), respectively.

This result is the anticipated operation plan of the hydropump plant, which must be communicated to the market in advance. This operation plan is changed in real time according to the real wind power production. This aspect will be explained and developed in Section 4.1.

In the joint operation program, not all the possible cases made in the simulations are covered, only $90 \%$ of them. However, if the coverage is increased up to $95 \%$ of the cases, an 
additional $18 \%$ capacity in the reservoir for overproduction and $26 \%$ in underproduction cases are required. This would limit the pump plant operation, and in some cases it might make the hedging program not feasible because the real dimensions of the reservoir would not be enough to cover the possible imbalances of the wind farm.

\section{Results}

\subsection{Real Operation of a Hydro-Pump Plant Compensating the imbalances of a Wind Power Producer}

The proposed strategy to cover the imbalances is tested by using real production data and forecasts for a day, verifying if the production program of the hydro-pump plant covers the power imbalances of the wind farm. All the experiments in

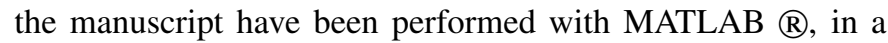
computer with processor Pentium Dual Core $3 \mathrm{GHz}$ with $3 \mathrm{~GB}$ memory.

In Fig. 8(a) the differences in the stored energy are shown. For this particular case, the total energy imbalances were predominantly positive, which means that there was an overproduction all the day, but some periods of negative errors, or underproduction also occurred.

Overproduction was used to store energy in the reservoir, as shown in Fig. 8(b), from the beginning of the operation until the end of hour 5, and then in hours 11 and 24, where it is possible to observe the operation of pumping due to the overproduction of wind power. On the other hand, there is a reduction in the pumping operation between hour 6 and until the end of the hour 7, where underproduction takes place.

In Fig. 8(c) the change in the hydro-pump plant program can be observed, when the plant operates as a generator. In this case, the underproduction is compensated, between hours 12 and 16. Then, the hydro generation was reduced due to wind power overproduction in the period between hours 17 and 23 .

\subsection{Anticipated and Actual Operations Cost}

The cost is calculated taking into account the dimensions of the wind power producer, and the hydro-pump plan. The prices of the market were taken from the Iberian market [27], as well as the reserve prices, for the day 2009/01/08 corresponding to the measurements and the predictions.

The revenue of the hydro-pump plant in the first case, the independent operation, is $4,375[€]$. In the second case, the joint operation, the revenue of the hydro-pump plant is $3,097[€]$, $29.2 \%$ less than in the independent operation. The difference between these two quantities is called Opportunity Cost, since it is the amount of money that the hydro-pump plant does not receive when it covers the power imbalances by modifying its original program.

The hydro-pump plant revenues obtained with the new modified program are lower, due to not being able to operate at full capacity. However, the real revenues will be calculated after the real operation of the plant, taking into account the power generated by the wind producer and the compensation actions of the hydro-pump plant, i.e. load consumption when operating as a pump and power generated by discharging the water of the reservoir.

The real revenue of the wind energy producer after the operation must be calculated considering the reserve price in the market. In the present case, these prices are taken from the MIBEL [27], a percentage of the energy hourly price in the same day. The cost of the imbalance is evaluated as follows:

$$
\sum_{i=1}^{n}\left(c_{i} \hat{P}_{\mathrm{W} i}+c_{i} d\right)
$$

where:

$$
d= \begin{cases}c_{i}^{\text {sell }}\left(P_{\mathrm{W} i}-\hat{P}_{\mathrm{W} i}\right) & \text { if } P_{\mathrm{W} i}>\hat{P}_{\mathrm{W} i}, \\ c_{i}^{\text {buy }}\left(\hat{P}_{\mathrm{W} i}-P_{\mathrm{W} i}\right) & \text { if } P_{\mathrm{W} i}<\hat{P}_{\mathrm{W} i}\end{cases}
$$

Finally, the real revenue of the hydro-pump plant and the wind power producer is estimated jointly from the unique bid of the wind power producer and the hydro-pump plant.

$$
\sum_{i=1}^{n}\left(c_{i} \bar{P}_{\mathrm{WHP} i}+c_{i} d\right)
$$

where:

$$
d= \begin{cases}c_{i}^{\text {sell }}\left(P_{\mathrm{WHP} i}-\bar{P}_{\mathrm{WHP} i}\right) & \text { if } P_{\mathrm{WHP} i}>\bar{P}_{\mathrm{WHP} i}, \\ c_{i}^{\text {buy }}\left(\bar{P}_{\mathrm{WHP} i}-P_{\mathrm{WHP} i}\right) & \text { if } P_{\mathrm{WHP} i}<\bar{P}_{\mathrm{WHP} i}\end{cases}
$$

with:

$$
\begin{aligned}
& \bar{P}_{\mathrm{WHP} i}=\hat{P}_{\mathrm{W} i}+\bar{P}_{\mathrm{H} i}-\bar{P}_{\mathrm{P} i} \\
& P_{\mathrm{WHP} i}=P_{\mathrm{W} i}+P_{\mathrm{H} i}-P_{\mathrm{P} i}
\end{aligned}
$$

The revenue of the Joint Operation is influenced for many variables such as: energy price, cost of the deviation, prices of other energy sources, SO requirements, or weather. For this reason two different sets of days with different wind production and price profiles have been taken in order to have an idea of the overall performance of the proposed method.

The problem discussed in Section 4.1, is considered a case of medium power production with a price profile of a typical weekday. Additionally, different price profiles of day ahead and reserve energy, for Saturday and Sunday days, as seen in Fig. 10(a) and Fig. 11(a), have been studied, corresponding to the same week of the first case analyzed, days 08, 10 and 11 of January 2009, as an example of a typical winter week. Also, two cases of production, high and low wind power production, are analyzed and compared, as seen in Fig. 9(a) and 9(b) respectively; these cases were chosen taking into account the power level during the day, high and low, and do not correspond to the price profile dates, but illustrate typical conditions. The revenues are compared before and after the real operation, both independently and jointly. Results are shown in Table 1.

As above, with the same set of wind power production, medium, high and low. But taking a different set of energy price profiles of day ahead and reserve, for weekday, Saturday and Sunday days, as seen in Fig. 10(b) and Fig. 11(b), have been studied, corresponding to the days 13, 15 and 16 of August 2009, as an example of a typical summer week, which is 

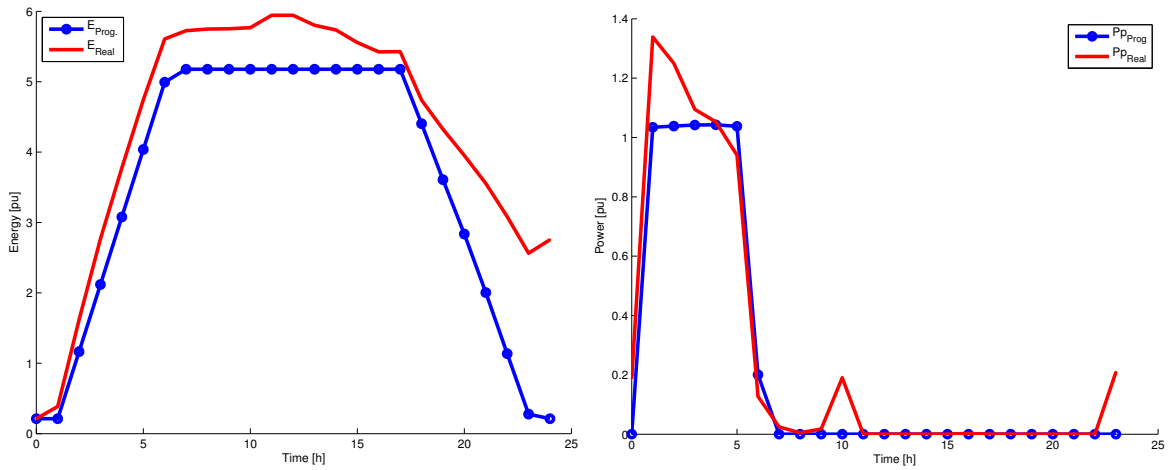

(a) Energy level in the reservoir, scheduled and real. (b) Power consumed by the pumping, scheduled and real.

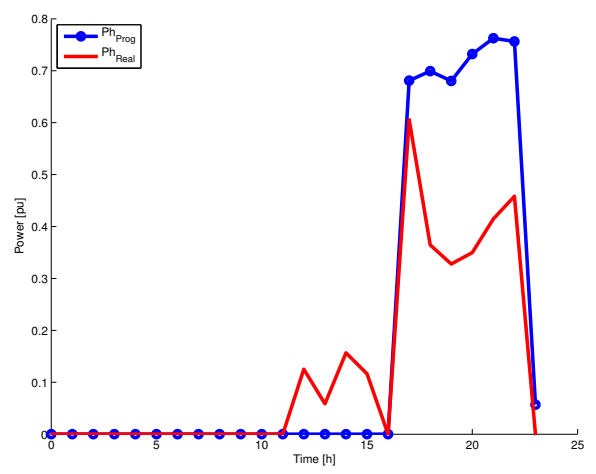

(c) Power generated, scheduled and real.

Figure 8: Comparison of the program obtained in advance and actual operation.

a period of low demand, as opposite of the winter case. The revenues are compared before and after the real operation, both independently and jointly. Results are shown in Table 2.

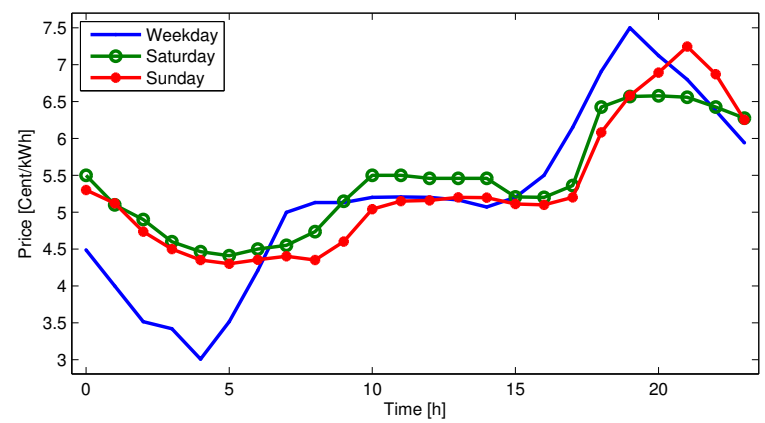

(a) Winter.

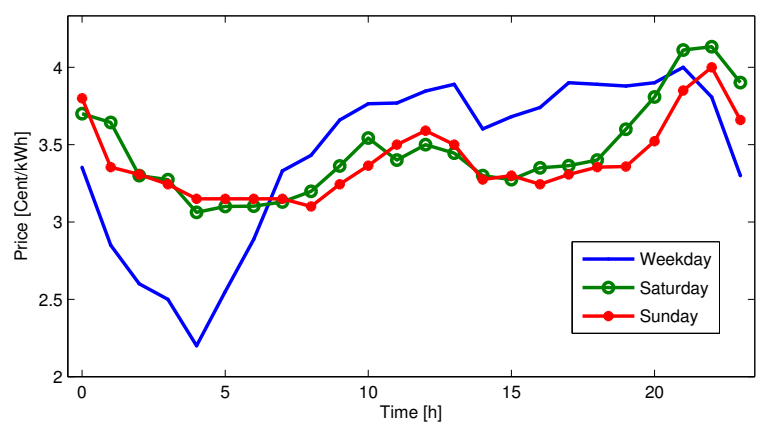

(b) Summer.

Figure 10: Price profiles types for typical days in a week.

Tables 1 and 2, has nine columns, clustered in groups of three. Each of these groups represents typical day prices: weekday, Saturday and Sunday respectively, and for one day, there are three types of wind production: medium, high and low, respectively. The "I" represents the independent operation of the utilities and "J" the joint operation.

The "Hydro-Pump Revenues (I)" represents the revenues that the hydro-pump plant obtains in the markets for the independent

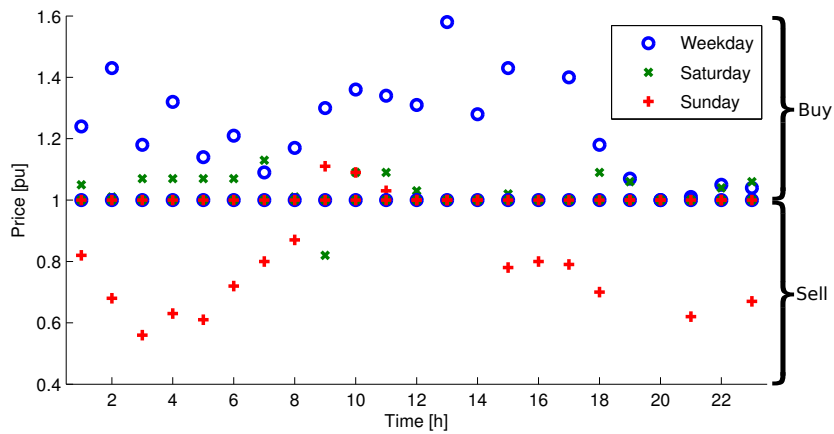

(a) Winter

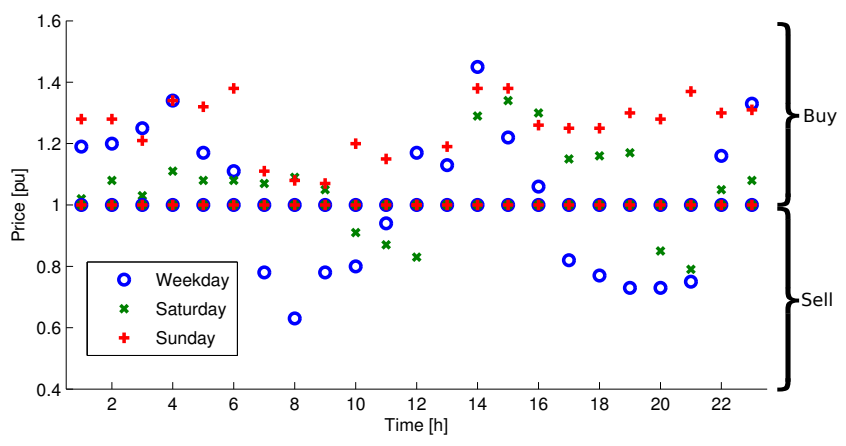

(b) Summer.

Figure 11: Deviation price profiles for typical days in a week.

operation, due to the optimal program. The "Wind Revenues (I)" represent the revenues that the wind producer (operating independently) obtains at the end of the operation, calculated by equations (24) and (25), in which case the wind power producer pays the imbalances. The "Total Revenues (I)" is the addition of the revenues of the two agents, operating independently.

The "Hydro-Pump Revenues (J)" represents the revenues obtained by the hydro-pump plant, when it modifies the initial program, independent operation, in order to cover the imbalance of the wind power producer in the future. With this value and the "Hydro-Pump Revenues (I)", the "Opportunity Cost" 


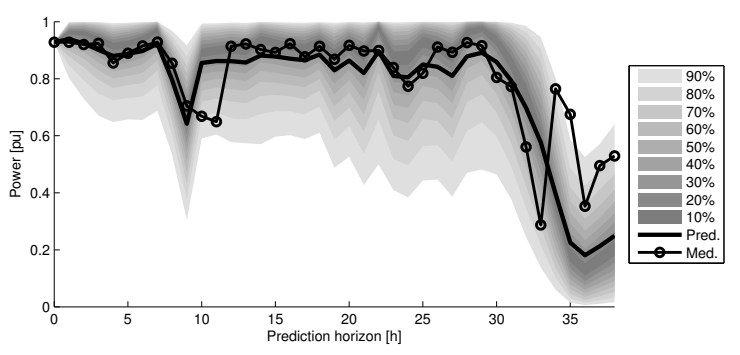

(a) High power production case.

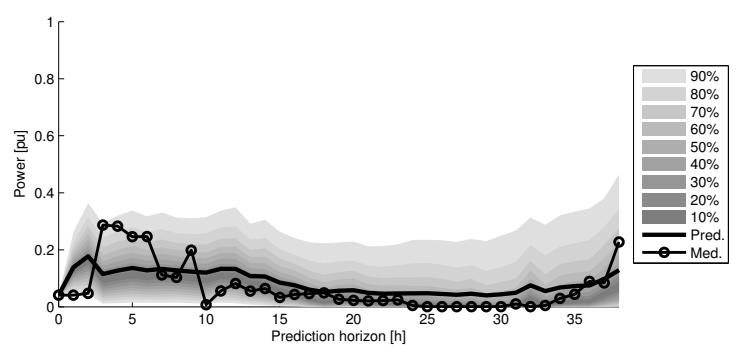

(b) Low power production case.

Figure 9: Possible scenarios, production and prediction of wind power based on the simulation.

\begin{tabular}{l|rrr|rrr|rrr}
\hline Day & \multicolumn{3}{|c|}{ weekday } & \multicolumn{3}{c|}{ Saturday } & \multicolumn{3}{c}{ Sunday } \\
\hline Power production & Medium & High & Low & Medium & High & Low & Medium & High & Low \\
\hline Hydro-Pump revenue (I) [€] & 4,375 & 4,375 & 4,375 & 1,446 & 1,446 & 1,446 & 2,209 & 2,209 & 2,209 \\
Wind revenue (I) [€] & 17,888 & 27,441 & 5,263 & 18,348 & 29,316 & 5,696 & 17,333 & 28,115 & 5,542 \\
Total revenue (I) [€] & 22,263 & 31,816 & 9,638 & 19,794 & 30,762 & 7,142 & 19,542 & 30,324 & 7,750 \\
& & & & & & & & & \\
Hydro-Pump revenue $(\mathrm{J})[€]$ & 3,097 & 3,419 & 3,744 & 991 & 1,172 & 1,231 & 1,466 & 1,779 & 1,898 \\
Opportunity Cost [€] & $-1,279$ & -956 & -631 & -455 & -274 & -214 & -743 & -430 & -311 \\
& $-29.22 \%$ & $-21.9 \%$ & $-14.4 \%$ & $-31.4 \%$ & $-18.9 \%$ & $-14.8 \%$ & $-33.6 \%$ & $-19.4 \%$ & $-14.1 \%$ \\
& & & & & & & & & \\
Real revenue (J) [€] & 24,419 & 36,627 & 18,057 & 23,646 & 37,076 & 16,066 & 23,465 & 35,814 & 15,644 \\
Revenue Difference [€] & 2,156 & 4,811 & 8,419 & 3,852 & 6,314 & 8,925 & 3,923 & 5,490 & 7,894 \\
& $9.7 \%$ & $15.1 \%$ & $87.3 \%$ & $19.5 \%$ & $20.5 \%$ & $125.0 \%$ & $20.1 \%$ & $18.1 \%$ & $101.9 \%$ \\
\hline
\end{tabular}

Table 1: Hydro-pump plant and wind power producer operation cost and revenues, winter.

\begin{tabular}{l|rrr|rrr|rrr}
\hline Day & \multicolumn{3}{|c|}{ weekday } & \multicolumn{3}{c|}{ Saturday } & \multicolumn{3}{c}{ Sunday } \\
\hline Power production & Medium & High & Low & Medium & High & Low & Medium & High & Low \\
\hline Hydro-Pump revenue (I) & 1,231 & 1,231 & 1,231 & 157 & 157 & 157 & 31 & 31 & 31 \\
Wind revenue (I) & 10,817 & 18,374 & 3,364 & 11,183 & 18,734 & 3,489 & 10,943 & 18,561 & 3,316 \\
Total revenue (I) & 12,048 & 19,605 & 4,595 & 11,340 & 18,891 & 3,646 & 10,974 & 18,592 & 3,347 \\
& & & & & & & & & \\
Hydro-Pump revenue (J) & 898 & 987 & 1,030 & -72 & 123 & 134 & -334 & -127 & -97 \\
Opportunity Cost & -333 & -244 & -201 & -229 & -34 & -23 & -365 & -157 & -128 \\
& $-27.0 \%$ & $-19.8 \%$ & $-16.4 \%$ & $-145.5 \%$ & $-21.8 \%$ & $-14.7 \%$ & $-1185.9 \%$ & $-511.6 \%$ & $-416.9 \%$ \\
& & & & & & & & & \\
Real revenue (J) & 15,301 & 23,780 & 10,731 & 13,582 & 20,942 & 6,327 & 14,672 & 21,854 & 6,947 \\
Revenue Difference & 3,253 & 4,175 & 6,136 & 2,242 & 2,051 & 2,681 & 3,698 & 3,262 & 3,601 \\
& $27.0 \%$ & $21.3 \%$ & $133.5 \%$ & $19.8 \%$ & $10.9 \%$ & $73.5 \%$ & $33.7 \%$ & $17.5 \%$ & $107.6 \%$ \\
\hline
\end{tabular}


for the hydro-pump plant is calculated, representing the losses in the plant revenues for modifying its original operating point. This value is given in Euros and in a percentage.

The "Real Revenues (J)", are the revenues obtained by the hydro-pump plant and wind power producer operating jointly; this value is calculated with the equations (26)-(29) at the end of the day analyzed, when the imbalances were covered.

Finally, "Revenues Difference" is the relationship between the "Total Revenues" of the hydro-pump plant and the wind power producer, operating independently and jointly. This value is given in money and in percentage.

The obtained results showed in Table 1 and 2, are very promising, since for the cases studied, the total revenues of the hydro-pump plant and the wind power producer operating jointly are positive, and an extra benefit is obtained when the imbalances in the prediction are covered. Additionally, in all the cases, the "Opportunity Costs" are less than the "Revenue Difference".

In Table 2, the "Hydro-Pump Revenues (J)" has negative values in some cases. This means that is more profitable to buy energy and to store this as water, and thus to ensure sufficient reserves to cover the possibles imbalances. These values are due to the shape of the energy profile, that is too flat, compared with the winter case. It becomes more difficult in this situation to obtain a benefit from the combined operation, compared with the winter prices when low and high periods are more pronounced. Therefore, in the simulated cases the joint operation is more profitable than the independent operation. The obtained revenues for the operation in cooperation must be later distributed between the wind farm and the hydro-pump plant.

\section{Conclusions}

In this paper, a hydro-pump plant is used to minimize the imbalances due to errors in the wind power forecasting when participating in an electricity market. In this way, the utilities may reduce the risk due to the uncertainty in the wind power prediction and in prices of the reserve market. Eventually, the imbalances in the wind power production could be fully avoided by the action of the hydro-pump station.

In order to deal with the uncertain behavior of the wind power production, a method to simulate scenarios of power production is proposed. The main feature of the proposed method is that the simulated scenarios have similar properties to the real data. In particular, they are able to reproduce the non-linear and non-gaussian behavior of wind power production. Besides, the proposed method has low computational requirements.

The calculation of the simulated scenarios is very promising because possible energy imbalance and the prediction errors intervals can be obtained, making easy to understand the behavior of wind energy production and the relationship with its prediction.

The statistical information obtained with the simulated scenarios makes it possible to calculate the joint optimal operation of a hydro-pump plant and a wind power producer. The results show economic gains for the operation in cooperation. The procedure can be expanded to independent control centers for renewable energy, allowing the design of investment strategies and some other operational issues.

The method uses both an optimization procedure and an estimation of the wind power uncertainty. The resulting strategies do not guarantee more benefits, but certainly have advantages, increasing the dynamic of the utility into the energy market. There are also advantages for the power system operation, because the utility could also control the voltage and frequency and store energy, making the system safer and enhancing the integration of renewable sources. An extensions of these strategies could also be applied in the next generation networks called Smart Grids.

\section{Acknowledgment}

The authors thanks for the financial support granted by the Ministerio de Ciencia e Innovación of Spain, IREMEL project with reference ENE2010-16074, called "Integración de Energías Renovables en el Mercado de Electricidad" and ANEMOS.plus and Integrated Action IT2009-0063 projects.

\section{References}

[1] M. Lei, L. Shiyan, J. Chuanwen, L. Hongling, Z. Yan, A review on the forecasting of wind speed and generated power, Renewable and Sustainable Energy Reviews 13 (2009) 915-920.

[2] R. Doherty, M. O'Malley, A new approach to quantify reserve demand in systems with significant installed wind capacity, IEEE Transactions on Power Systems 20 (2005) 587-595.

[3] G. Koeppel, M. Korpås, Increasing the network in-feed accuracy of wind turbines with energy storage devices, in: The Sixht World Energy System Conference, Torino, Italy.

[4] J. Matevosyan, L. Söder, Short-term hydropower planning coordinated with wind power in areas with congestion problems, Wind Energy 10 (2007) 195-208.

[5] H. Holttinen, J. Ikäheimo, Wind prediction and bids in Denmark, Technical Report, VTT Energia, 2007.

[6] P. Pinson, H. Madsen, H. A. Nielsen, G. Papaefthymiou, B. Klöckl, From probabilistic forecasts to statistical scenarios of short-term wind power production, Wind Energy 12 (2009) 51-62.

[7] P. Pinson, P. George, K. Bernd, V. Jody, Dynamic sizing of energy storage for hedging wind power forecast uncertainty, in: IEEE Power \& Energy Society General Meeting, Calgary, Canada.

[8] E. D. Castronuovo, J. A. P. Lopes, On the optimization of the daily operation of a wind-hydro power plant, IEEE Transactions on Power Systems 19 (2004) 1599-1606.

[9] E. D. Castronuovo, J. A. P. Lopes, Optimal operation and hydro storage sizing of a wind-hydro power plant, International Journal of Electrical Power \& Energy Systems 26 (2004) 771-778.

[10] M. A. Ortega-Vazquez, D. S. Kirschen, Estimating the spinning reserve requirements in systems with significant wind power generation penetration, IEEE Transactions on Power Systems 24 (2009) 114-124.

[11] C. Abbey, G. Joos, A stochastic optimization approach to rating of energy storage systems in Wind-Diesel isolated grids, IEEE Transactions on Power Systems 24 (2009) 418-426.

[12] J. García-González, R. de la Muela, L. Santos, A. Gonzalez, Stochastic joint optimization of wind generation and Pumped-Storage units in an electricity market, IEEE Transactions on Power Systems 23 (2008) 460468.

[13] J. M. Crespo, J. L. Angarita, E. D. Castronuovo, H. Amaris, J. Usaola, Tools for the effective integration of large amounts of wind energy in the system, in: E. D. Castronuovo (Ed.), Optimization Advances in Electric Power Systems, Nova Science Publishers, New York, USA, 2008. 
[14] J. M. Angarita, J. G. Usaola, Combining hydro-generation and wind energy biddings and operation on electricity spot markets, Electric Power Systems Research 77 (2007) 393-400.

[15] D. X. Li, On default correlation, The Journal of Fixed Income 9 (2000) 43-54.

[16] R. B. Nelsen, An Introduction to Copulas, Springer, New York, USA, 1 edition, 1998

[17] Álvaro Jaramillo, I. Sánchez, E. Castronuovo, J. Usaola, Simulating scenarios and prediction intervals in wind power forecasting with the beta distribution, in: 11th. Spanish-Portuguese Conference on Electrical Engineering (11CHLIE), Zaragoza, Spain, p. 5.

[18] P. Pinson, H. A. Nielsen, J. K. Møller, H. Madsen, G. N. Kariniotakis, Non-parametric probabilistic forecasts of wind power: required properties and evaluation, Wind Energy 10 (2007) 497-516.

[19] J. B. Bremnes, Probabilistic wind power forecasts using local quantile regression, Wind Energy 7 (2004) 47-54.

[20] J. Usaola, Probabilistic load flow with wind production uncertainty using cumulants and Cornish-Fisher expansion, International Journal of Electrical Power \& Energy Systems 31 (2009) 474-481.

[21] H. Bludszuweit, J. Dominguez-Navarro, A. Llombart, Statistical analysis of wind power forecast error, Power Systems, IEEE Transactions on 23 (2008) 983-991.

[22] A. Sklar, Fonctions de répartition à n dimensions et leurs marges, Publ. Inst. Statist. Univ. Paris 8 (1959) 229-23.

[23] R. D. Cook, S. Weisberg, Residuals and Influence in Regression, 1982.

[24] I. Sánchez, Short-term prediction of wind energy production, International Journal of Forecasting 22 (2006) 43-56.

[25] I. Sánchez, J. Usaola, O. Ravelo, C. Velasco, J. Domínguez, M. G. Lobo, G. González, F. Soto, B. Diaz-Guerra, M. Alonso, Sipreólico-a wind power prediction system based on flexible combination of dynamic models. application to the spanish power system, in: World Wind Energy Conference in Berlin, Germany.

[26] A. Conejo, M. Plazas, R. Espinola, A. Molina, Day-ahead electricity price forecasting using the wavelet transform and ARIMA models, IEEE Transactions on Power Systems 20 (2005) 1035-1042.

[27] Sistema de información del operador del sistema, Red Eléctrica de España, S. A., 2010. http://www.esios.ree.es/web-publica/. 